\title{
Non-Pilot-Aided Sequential Monte Carlo Method to Joint Signal, Phase Noise, and Frequency Offset Estimation in Multicarrier Systems
}

\author{
François Septier, ${ }^{1}$ Yves Delignon, ${ }^{2}$ Atika Menhaj-Rivenq, ${ }^{1}$ and Christelle Garnier ${ }^{2}$ \\ ${ }^{1}$ IEMN-DOAE UMR 8520, UVHC Le Mont Houy, 59313 Valenciennes Cedex 9, France \\ ${ }^{2}$ GET/INT/Telecom Lille 1, 59658 Villeneuve d'Ascq, France
}

Correspondence should be addressed to François Septier, francois.septier@telecom-lille1.eu

Received 27 July 2007; Accepted 2 April 2008

Recommended by Azzedine Zerguine

We address the problem of phase noise $(\mathrm{PHN})$ and carrier frequency offset $(\mathrm{CFO})$ mitigation in multicarrier receivers. In multicarrier systems, phase distortions cause two effects: the common phase error (CPE) and the intercarrier interference (ICI) which severely degrade the accuracy of the symbol detection stage. Here, we propose a non-pilot-aided scheme to jointly estimate $\mathrm{PHN}, \mathrm{CFO}$, and multicarrier signal in time domain. Unlike existing methods, non-pilot-based estimation is performed without any decision-directed scheme. Our approach to the problem is based on Bayesian estimation using sequential Monte Carlo filtering commonly referred to as particle filtering. The particle filter is efficiently implemented by combining the principles of the RaoBlackwellization technique and an approximate optimal importance function for phase distortion sampling. Moreover, in order to fully benefit from time-domain processing, we propose a multicarrier signal model which includes the redundancy information induced by the cyclic prefix, thus leading to a significant performance improvement. Simulation results are provided in terms of bit error rate (BER) and mean square error (MSE) to illustrate the efficiency and the robustness of the proposed algorithm.

Copyright (c) 2008 François Septier et al. This is an open access article distributed under the Creative Commons Attribution License, which permits unrestricted use, distribution, and reproduction in any medium, provided the original work is properly cited.

\section{INTRODUCTION}

Multicarrier transmission systems have aroused great interest in recent years as a potential solution to the problem of transmitting high data rate over a frequency selective fading channel [1]. Today, multicarrier modulation is being selected as the transmission scheme for the majority of new communication systems [2]. Examples include digital subscriber line (DSL), European digital video broadcast (DVB), digital audio broadcast (DAB), and wireless local area network (WLAN) standards (IEEE 802.11 and 802.16).

However, multicarrier systems are very sensitive to phase noise (PHN) and carrier frequency offset (CFO) caused by the oscillator instabilities [3-8]. Indeed, random time-varying phase distortions destroy the orthogonality of subcarriers and lead after the discrete Fourier transform (DFT) both to rotation of every subcarrier by a random phase, called common phase error (CPE), and to intercarrier interference (ICI).
In literature, many approaches have been proposed to estimate and compensate PHN in OFDM systems either in the time domain [9] or in the frequency domain [10-15]. All these methods require the use of pilot subcarriers in each OFDM symbol which limits the system spectral efficiency. Non-pilot-aided estimation algorithms are, therefore, a challenging task since they have the advantage of being bandwidth more efficient. Such methods have already been proposed to compensate phase distortions $[16,17]$ in OFDM systems. In [16], the authors propose an interesting CPE correction scheme. However, when phase distortions become significant, this approach has limited performances as it neglects ICI. Recently in [17], a joint data and PHN estimator via variational inference approach has been proposed using the small PHN assumption. Moreover, in algorithms [16, 17], a decision-directed scheme is used at the initialization step in order to make a tentative decision over the transmitted symbols without any phase distortion correction. Consequently, for significant phase distortions, noise-induced 
symbol decision errors may propagate through the feedback loop, leading to poor estimator performance.

In this paper, we propose a new non-pilot-aided algorithm for joint PHN, CFO, and signal estimation in general multicarrier systems without any decision-directed scheme. Because of the nonlinear behavior of the received signal, we use a simulation-based recursive algorithm from the family of sequential Monte Carlo (SMC) methods also referred to as particle filtering [18]. Sequential Monte Carlo methodology is useful to prevent the sampling dimension from blowing up with time (with the number of subcarriers in our context), unlike classical offline stochastic methods such as Monte Carlo methods or also Markov chain Monte Carlo (MCMC) methods. Furthermore, since statistical a priori information about time evolution of phase distortions is known, estimation is carried out in the time domain. Time domain processing allows taking into account the redundancy information induced by the cyclic prefix, yielding an efficient algorithm.

This paper is organized as follows. In Section 2, both the phase distortions and the multicarrier system model are described. In Section 3, the suggested observation and state equations are defined leading to the dynamic state-space (DSS) representation. In Section 4, we review fundamentals of particle filter and describe the proposed marginalized particle filter algorithm using an approximate optimal importance function. The posterior Cramér-Rao bound (PCRB) which corresponds to the lowest bound achieved by the optimal estimator is also derived in this section. In Section 5, numerical results are given to demonstrate the validity of our approach. The efficiency and the robustness of the proposed non-pilot-aided marginalized particle filter algorithm are assessed for both OFDM and MC-CDMA systems and are compared to existing schemes. Finally, Section 6 presents some concluding remarks.

\section{PROBLEM FORMULATION}

In this paper, $N, N_{\mathrm{cp}}$, and $T$ denote, respectively, the number of subcarriers, the cyclic prefix length, and the OFDM symbol duration excluding the cyclic prefix. Let $\mathcal{N}(x ; \mu, \boldsymbol{\Sigma})$ and $\mathcal{N}_{c}(x ; \mu, \Sigma)$ represent, respectively, real and circularly symmetric complex Gaussian random vectors with mean $\mu$ and covariance matrix $\boldsymbol{\Sigma}$. $\mathbf{I}_{n}$ and $\mathbf{0}_{n \times m}$, are respectively, the $n \times n$ identity matrix and the $n \times m$ matrix of zeros. Finally, lower case bold letters are used for column vectors and capital bold letters for matrices; $(\cdot)^{*},(\cdot)^{T}$ and $(\cdot)^{H}$ denote, respectively, conjugate, transpose, and Hermitian transpose.

\subsection{Phase distortion model}

In a baseband complex equivalent form, the carrier delivered by the noisy oscillator can be modeled as

$$
p(t)=\exp (j \phi(t))
$$

where the phase distortion $\phi(t)$ represents both the phase noise $(\mathrm{PHN})$ and the carrier frequency offset $(\mathrm{CFO})$ and can be written as follows:

$$
\phi(t)=\theta(t)+2 \pi \Delta f t
$$

where $\theta(t)$ and $\Delta f$ correspond, respectively, to the $\mathrm{PHN}$ and the CFO. The PHN is modeled as a Brownian process $[3,5]$. The power spectral density of $\exp (\theta(t))$ has a Lorentzian shape controlled by the parameter $\beta$ representing the two-sided $3 \mathrm{~dB}$ bandwidth. This model produces a $1 / f^{2}$ type noise power behavior that agrees with experimental measurements carried out on real RF oscillators. The phase noise rate is characterized by the bandwidth $\beta$ normalized with respect to the OFDM symbol rate $1 / T$, namely, by the parameter $\beta T$. At the sampling rate of the receiver $N / T$, the discrete form of the PHN, for $k=0, \ldots, N+N_{\text {cp }}-1$, is

$$
\theta_{n, k}=\theta_{n, k-1}+v_{n, k}
$$

where $k$ denotes the $k$ th sample, $n$ the $n$th OFDM symbol and $v_{n, k}$ is an independent and identically distributed (i.i.d.) zero mean Gaussian variable with variance $\sigma_{v}^{2}=2 \pi \beta T / N$.

Finally, using (2) and (3) and assuming the initial condition $\phi_{n,-1}=0$ as in $[5,17]$, a discrete recursive relation for the phase distortions is obtained

$$
\phi_{n, k}= \begin{cases}v_{n, 0}, & \text { if } k=0 \\ \phi_{n, k-1}+\frac{2 \pi \epsilon}{N}+v_{n, k}, & \text { otherwise }\end{cases}
$$

where $\epsilon=\Delta f T$ is the normalized CFO with respect to the subcarrier spacing.

\subsection{Multicarrier system model}

Figure 1 shows the block diagram of a downlink multicarrier system. The MC-CDMA includes the OFDM modulation when the spreading code length $L_{c}=1$ and the number of users $N_{u}=1$. So below, the index $u$ which corresponds to the $u$ th user is omitted for the OFDM system. For simplicity, in the case of MC-CDMA, $L_{c}$ is chosen equal to $N$.

First, for each user $u=1 \cdots N_{u}$, the input i.i.d. bits are encoded into M-QAM symbols $X_{i}^{u}$ which are assumed to form an i.i.d. zero mean random process with unitary power. Then after the inverse discrete Fourier transform (IDFT), the samples of the transmitted signal can be written as

$$
s_{n, l}=\frac{1}{\sqrt{N}} \sum_{i=0}^{N-1} d_{n, i} e^{j 2 \pi i l / N}
$$

Whatever the multicarrier system, $s_{n, l}$ can be viewed as an OFDM symbol, where $l$ denotes the $l$ th sample and $n$ the $n$th OFDM symbol. Only $d_{n, i}$ differs according to the multicarrier system:

$$
d_{n, i}= \begin{cases}X_{n N+i}, & \text { for OFDM system, } \\ \sum_{u=1}^{N_{u}} X_{n}^{u} c_{i}^{u}, & \text { for MC-CDMA system, }\end{cases}
$$

where $\left\{c_{k}^{u}\right\}_{k=0}^{L_{c}-1}$ represents the spreading code of the $u$ th user. Equation (5) also holds for the MC-DS-CDMA 
system, which is not treated in this paper, with $d_{n, i}=$ $\sum_{u=1}^{N_{u}} X_{\lfloor n / N\rfloor N+i}^{u} c_{n \bmod L_{c}}^{u}$, where the operator $\lfloor\cdot\rfloor$ stands for the largest integer smaller than or equal to $(\cdot)$.

The general OFDM symbol defined in (5) is extended with a cyclic prefix of $N_{c p}$ samples, larger than the channel maximum excess delay in order to prevent interference between adjacent OFDM symbols. The resulted signal forms the transmitted signal (Figure 2).

The time varying frequency selective channel $h(t, \tau)$ is assumed to be static over several OFDM symbols. Assuming perfect time synchronization, the $n$th received OFDM symbol can be expressed as shown in (7), where vectors $\mathbf{r}_{\mathbf{n}}$, $\tilde{\mathbf{s}}_{\mathbf{n}}, \mathbf{w}_{\mathbf{n}}$, and matrices $\boldsymbol{\Phi}_{\mathbf{n}}, \boldsymbol{\Omega}_{\mathbf{n}}$ have the following respective sizes $\left(N+N_{\mathrm{cp}}\right) \times 1,\left(N+N_{\mathrm{cp}}+L-1\right) \times 1,\left(N+N_{\mathrm{cp}}\right) \times 1$, $\left(N+N_{\mathrm{cp}}\right) \times\left(N+N_{\mathrm{cp}}\right),\left(N+N_{\mathrm{cp}}\right) \times\left(N+N_{\mathrm{cp}}+L-1\right):$
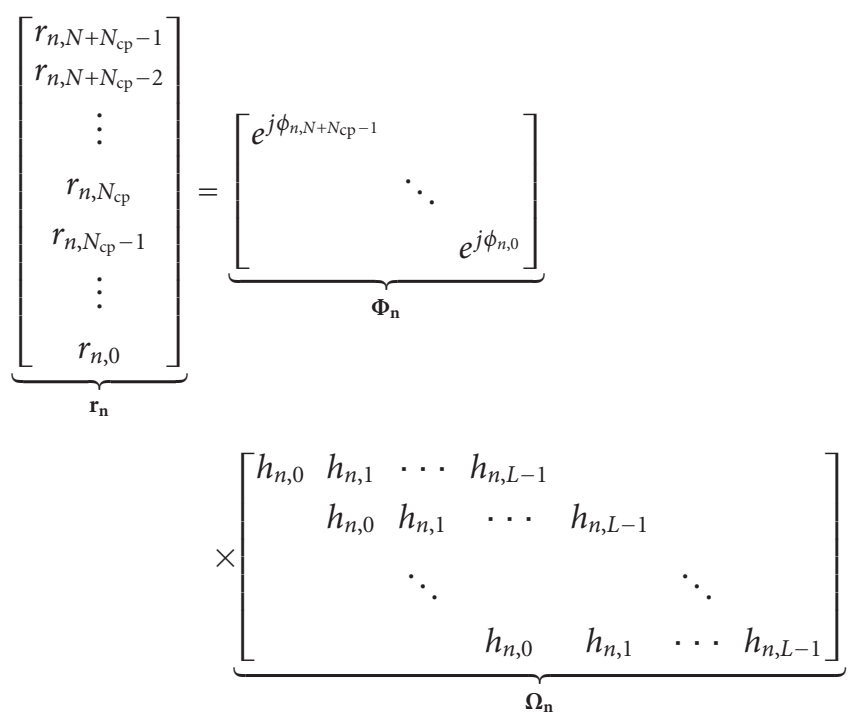

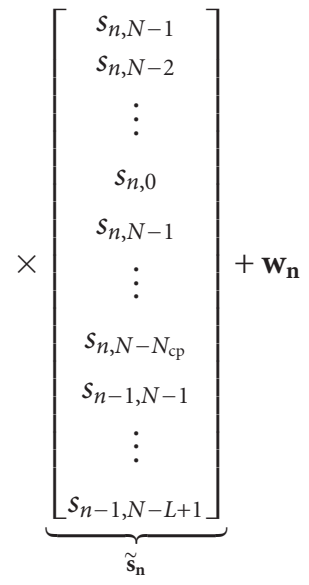

$\Phi_{\mathbf{n}}$ and $\mathbf{w}_{\mathbf{n}}$, correspond, respectively, to the phase distortions and to the additive white Gaussian noise:

$$
\mathbf{w}_{\mathbf{n}}=\left[w_{n, N+N_{\mathrm{cp}}-1} \cdots w_{n, 0}\right]^{\mathbf{T}},
$$

where each element $w_{n, l}$ is a circular zero-mean white Gaussian noise with power $\sigma_{w}^{2}$.

After discarding the cyclic prefix and performing discrete Fourier transform (DFT) on the remaining $N$ samples (i.e., $\left.r_{n, N_{\text {cp }}}, \ldots, r_{n, N_{\text {cp }}+N-1}\right)$, it is straightforward to show that the demodulated signal at subcarrier $k$ depends on all the phase distortion states $\left\{\phi_{n, N_{\text {cp }}}, \ldots, \phi_{n, N_{\text {cp }}+N-1}\right\}$ leading to the CPE and the ICI $[3,6]$. In contrast, we remark that each observation $r_{n, k}(7)$ depends on only one phase distortion state $\phi_{n, k}$. Therefore, using the statistical a priori knowledge of phase distortion time evolution (4), the tracking ability of time-domain methods appears as a promising alternative to frequency domain schemes for phase distortion mitigation in multicarrier systems.

\section{PHASE DISTORTION MITIGATION}

In this paper, we propose a new robust non-pilot-aided scheme to jointly estimate in the time domain both the transmitted signal and the phase distortions. After demodulating the estimate of the transmitted multicarrier signal by the FFT transform, symbol detection is carried out without any additional frequency equalizer. The mathematical foundation of our solution is the Bayesian theory. Its use requires a dynamic state-space system (DSS) which includes both state and measurement equations. In the first part of this section, the measurement equation is derived. The two state processes are given by the phase distortions and by the transmitted signal. The state equation of the phase distortions is directly defined by (4). Consequently, only the state equation of the multicarrier signal is derived in the second part of this section. Finally, these equations lead to the definition of the DSS model.

\subsection{Observation equation}

Using (7), $r_{n, k}$ can be written as follows:

$$
r_{n, k}=e^{j \phi_{n, k}} \sum_{l=0}^{L-1} h_{n, l} s_{n, k-N_{\mathrm{cp}}-l}+w_{n, k},
$$

where $w_{n, k}$ is a circular zero-mean Gaussian noise with variance $\sigma_{w}^{2}$. This observation equation takes into account both the insertion of the cyclic prefix and the interference intersymbol (ISI) due to the multipath channel. We use the following definition of $s_{n, k}$ for $k<0$ :

$$
s_{n, k}= \begin{cases}s_{n, N+k}, & \text { if }-N_{\mathrm{cp}} \leq k \leq-1, \\ s_{n-1, k+N+N_{\mathrm{cp}},} & \text { if } k<-N_{\mathrm{cp}} .\end{cases}
$$

In matrix form, (9) can be rewritten as

$$
r_{n, k}=e^{j \phi_{n, k}} \mathbf{h}_{n}^{T} \mathbf{s}_{n, k}+w_{n, k}
$$

with

$$
\begin{aligned}
\mathbf{h}_{n} & =\left[\begin{array}{llll}
h_{n, 0} & \cdots & h_{n, L-1} & \mathbf{0}_{1 \times\left(N+N_{\mathrm{cp}}-1\right)}
\end{array}\right]^{T}, \\
\mathbf{s}_{n, k} & =\left[\begin{array}{llll}
s_{n, k-N_{\mathrm{cp}}} & \cdots & s_{n,-N_{\mathrm{cp}}-L+1} & \mathbf{0}_{1 \times\left(N+N_{\mathrm{cp}}-k-1\right)}
\end{array}\right]^{T} .
\end{aligned}
$$

The observation equation (11) involves two unknown states: the CFO and the PHN included in $\phi_{n, k}$ and the transmitted multicarrier signal $\mathbf{s}_{n, k}$. The general objective is 


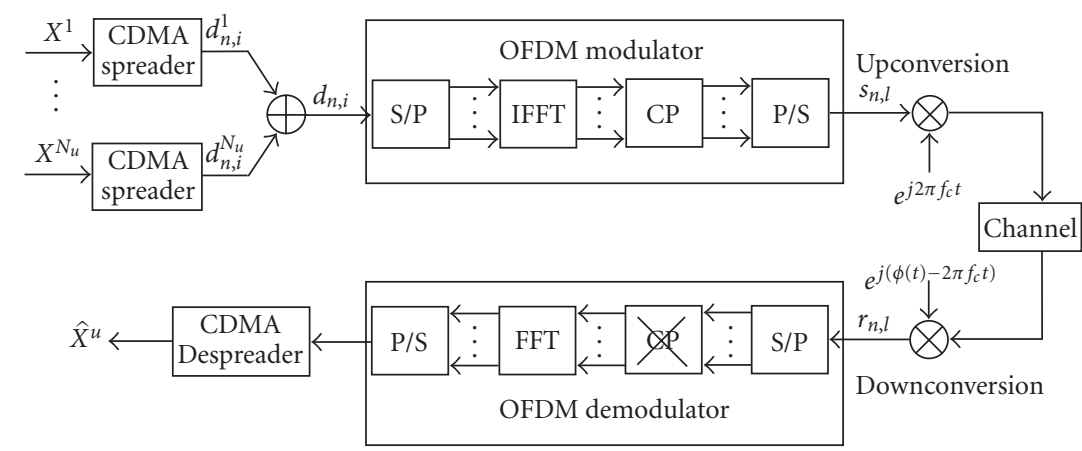

FIGURE 1: Block diagram of the transmission system including both MC-CDMA and OFDM systems.

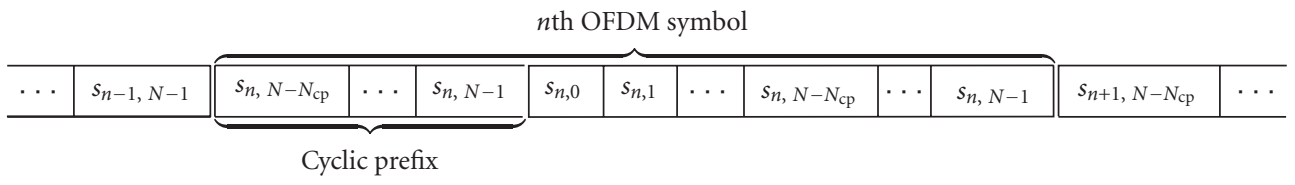

FIgURE 2: Example of a multicarrier system flow.

to jointly and adaptively estimate these two dynamic states using the set of received signals, $r_{n, k}$, with $k=0, \ldots, N+$ $N_{c p}-1$. Since the a priori dynamic feature of $\phi_{n, k}$ is already given by (4), only the state equation of $\mathbf{s}_{n, k}$ is required for the joint a posteriori estimation.

\subsection{State equation of the multicarrier signal}

The cyclic prefix in the multicarrier system is a copy of the last portion of the symbol appended to the front of the OFDM symbol, so that the multicarrier signal may be characterized as a cyclostationary process with period $N$. Using this property and the assumption of i.i.d., transmitted symbols with unitary power, $E\left[\left|s_{n, k}\right|^{2}\right]=1$, we derive the following relation:

$$
E\left[s_{n, k} s_{n, l}^{*}\right]
$$$$
= \begin{cases}1, & \text { if } k=l+z N \text { with } z \in \mathbb{Z}, k \in\left[-N_{\mathrm{cp}} ; N-1\right], \\ 0, & \text { otherwise, }\end{cases}
$$

where $\mathbb{Z}$ denotes the rational integer domain. According to the central limit theorem, the envelope of the multicarrier signal can be approximated by a circular Gaussian distributed random variable. The central limit theorem has been already used in literature to approximate the OFDM signal as a circularly Gaussian random vector, especially for the derivation of analytical expression of the peak-toaverage power ratio (PAPR) [19, 20]. In [21], a rigorous proof establishes that the complex envelope of a bandlimited uncoded OFDM signal converges weakly to a Gaussian random process. As shown in Appendix A, this approximation provides an accurate modeling of the multicarrier signal. Therefore, the state equation of the vector $\mathbf{s}_{n, k}$ is written in the matrix form as

$$
\mathbf{s}_{n, k}=\mathbf{A}_{n, k} \mathbf{s}_{n, k-1}+\mathbf{b}_{n, k}
$$

where the transition matrix $\mathbf{A}_{n, k}$ is defined as

$$
\mathbf{A}_{n, k}=\left[\begin{array}{lll} 
& \xi_{n, k}^{T} & \\
\mathbf{I}_{\left(N+N_{\mathrm{cp}}+L-2\right)} & & \mathbf{0}_{\left(N+N_{\mathrm{cp}}+L-2\right) \times 1}
\end{array}\right] .
$$

Using relation (13), $\xi_{\mathbf{n}, \mathbf{k}}$ is given by

$\xi_{\mathbf{n}, \mathbf{k}}= \begin{cases}{\left[\begin{array}{ll}\mathbf{0}_{1 \times\left(N+N_{\mathrm{cp}}+L-1\right)}\end{array}\right]^{T},} & \text { if } 0 \leq k \leq N-1, \\ {\left[\begin{array}{lll}\mathbf{0}_{1 \times(N-1)} & 1 & \mathbf{0}_{1 \times\left(N_{\mathrm{cp}}+L-1\right)}\end{array}\right]^{T},} & \text { if } N \leq k \leq N+N_{\mathrm{cp}}-1 .\end{cases}$

Finally, $\mathbf{b}_{n, k}$ is a circular $\left(N+N_{\mathrm{cp}}+L-1\right)$-by-1 zero-mean Gaussian noise vector with the following covariance matrix:

$$
E\left[\mathbf{b}_{n, k} \mathbf{b}_{n, k}^{H}\right]=\left[\begin{array}{cccc}
\sigma_{b_{n, k}}^{2} & 0 & \cdots & 0 \\
0 & 0 & & \vdots \\
\vdots & & \ddots & \vdots \\
0 & \cdots & \cdots & 0
\end{array}\right],
$$

where

$$
\sigma_{b_{n, k}}^{2}= \begin{cases}1, & \text { if } 0 \leq k \leq N-1 \\ 0, & \text { if } N \leq k \leq N+N_{\mathrm{cp}}-1\end{cases}
$$




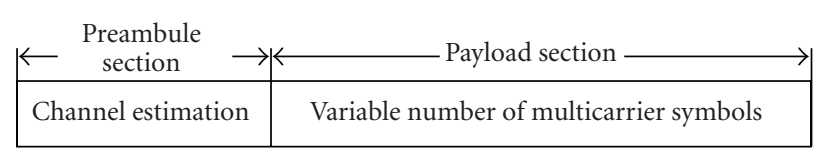

FIGURE 3: Multicarrier packet structure.

\subsection{Dynamic state space model (DSS)}

By using (4), (14), and (11), we obtain the following DSS model:

$$
\begin{aligned}
\phi_{n, k} & = \begin{cases}v_{n, 0}, & \text { if } k=0, \\
\phi_{n, k-1}+\frac{2 \pi \epsilon}{N}+v_{n, k}, & \text { if } k=1, \ldots, N+N_{\mathrm{cp}}-1\end{cases} \\
\mathbf{s}_{n, k} & =\mathbf{A}_{n, k} \mathbf{s}_{n, k-1}+\mathbf{b}_{n, k}, \\
r_{n, k} & =e^{j \phi_{n, k}} \mathbf{h}_{n}^{T} \mathbf{s}_{n, k}+w_{n, k} .
\end{aligned}
$$

In order to jointly estimate $\epsilon, \phi_{n, k}$, and $\mathbf{s}_{n, k}$, we need the joint posterior probability density function (p.d.f.) $p\left(\phi_{n, 0: k}, \epsilon, \mathbf{s}_{n, k} \mid r_{n, 0: k}\right)$. Unfortunately this $p . d . f$. is analytically intractable, so we propose to numerically approximate $p\left(\phi_{n, 0: k}, \epsilon, \mathbf{s}_{n, k} \mid r_{n, 0: k}\right)$ via particle filtering [18]. Let us note that we assume in this paper the perfect knowledge of both AWGN and PHN variances, that is, $\sigma_{v}^{2}$ and $\sigma_{w}^{2}$, and also the channel impulse response $\mathbf{h}$. In fact, most of standards based on multicarrier modulation such as Hiperlan2 or IEEE 802.11a include training symbols used to estimate the channel impulse response before the data transmission as illustrated by Figure 3. Indeed, since the CIR changes slowly (in Hiperlan/2 standard specifications, the channel variations are supposed to correspond to terminal speeds $v \leq 3 \mathrm{~m} / \mathrm{s}$.) with respect to the OFDM symbol rate, the channel is thus only estimated at the beginning of a frame. Then its estimates is used for data detection in the payload section. In [22], we have proposed a joint channel, phase distortions, and both AWGN and PHN variances using SMC methodology. So these estimates obtained from the training sequence remain valid in the payload section when dealing with slow-fading channel. For simplicity, we assume in this paper that their values are perfectly known by the receiver since we focus on the payload section and thus on the problem of data detection in multicarrier systems in the presence of phase distortions.

\section{PARTICLE FILTER}

\subsection{Introduction}

The maximum a posteriori estimation of the state from the measurement is obtained under the framework of the Bayesian theory which has been mainly investigated in Kalman filtering [23]. The Kalman filter is optimal when the state and measurement equations are linear and noises are independent, additive, and Gaussian. When these assumptions are not fulfilled, various approximation methods have been developed among which the extended Kalman filter is the most commonly used [23].
Since the nineties, particle filtering has become a powerful methodology to cope with nonlinear and non-Gaussian problems [24] and represents an important alternative to extended Kalman filter (EKF). The main advantage lies in an approximation of the distribution of interest by discrete random measures, without any linearization.

There are several variants of particle filters. The sequential importance sampling (SIS) algorithm is a Monte Carlo method that is the basis for most sequential Monte Carlo filters. The SIS algorithm consists in recursively estimating the required posterior density function $p\left(x_{0: k} \mid y_{0: k}\right)$ which is approximated by a set of $N$ random samples with associated weights, denoted by $\left\{x_{0: k}^{(m)}, w_{k}^{(m)}\right\}_{m=1 \cdots N}$ :

$$
\hat{p}\left(x_{0: k} \mid y_{0: k}\right)=\sum_{j=1}^{N} \delta\left(x_{0: k}-x_{0: k}^{(j)}\right) \widetilde{w}_{k}^{(j)}
$$

where $x_{k}^{(j)}$ is drawn from the importance function $\pi\left(x_{k}\right.$ $\left.x_{0: k-1}^{(j)}, y_{0: k}\right), \delta(\cdot)$ is the Dirac delta function and, $\widetilde{w}_{k}^{(j)}=$ $w_{k}^{(j)} / \sum_{m=1}^{N} w_{k}^{(m)}$ is the normalized importance weight associated with the $j$ th particle.

The weights $w_{k}^{(m)}$ are updated according the concept of importance sampling:

$$
w_{k}^{(m)} \propto \frac{p\left(y_{k} \mid x_{0: k}^{(m)}\right) p\left(x_{k}^{(m)} \mid x_{0: k-1}^{(m)}\right)}{\pi\left(x_{k}^{(m)} \mid x_{0: k-1}^{(m)}, y_{0: k}\right)} w_{k-1}^{(m)} .
$$

After a few iterations, the SIS algorithm is known to suffer from degeneracy problems. To reduce these problems, the sequential importance resampling (SIR) integrates a resampling step to select particles for new generations in proportion to the importance weights [18]. Liu and Chen [25] have introduced a measure known as effective sample size:

$$
N_{\mathrm{eff}}=\frac{1}{\sum_{m=1}^{N}\left(\widetilde{w}_{k}^{(m)}\right)^{2}}
$$

and have proposed to apply the resampling procedure whenever $N_{\text {eff }}$ goes below a predefined threshold. For the resampling step, we use the residual resampling scheme described in [26]. This scheme outperforms the simple random sampling scheme with a small Monte Carlo variance and a favorable computational time [27, 28].

\subsection{Joint multicarrier signal, CFO and PHN estimation using marginalized particle filter}

Previously, we have explained how particle filtering can be used to obtain the posterior density function $p\left(x_{0: k} \mid y_{0: k}\right)$. In the case of our DSS model (19), the state vector $x_{k}$ is defined as

$$
x_{k}=\left[\phi_{n, k}, \epsilon, \mathbf{s}_{n, k}\right]
$$

In order to provide the best approximation of the a posteriori p.d.f., we take advantage of the linear substructure 
contained in the DSS model. The corresponding variables are marginalized out and estimated using an optimal linear filter. This is the main idea behind the marginalized particle filter, also known as the Rao-Blackwellized particle filter $[18,29,30]$. Indeed, conditioned on the nonlinear state variable $\phi_{n, k}$, there is a linear substructure in (19). Using Bayes' theorem, the posterior density function of interest can thus be written as

$$
p\left(\phi_{n, 0: k}, \epsilon, \mathbf{s}_{n, k} \mid r_{n, 0: k}\right)=p\left(\mathbf{s}_{n, k} \mid \phi_{n, 0: k}, r_{n, 0: k}\right) p\left(\phi_{n, 0: k}, \epsilon \mid r_{n, 0: k}\right),
$$

where $p\left(\mathbf{s}_{n, k} \mid \phi_{n, 0: k}, r_{n, 0: k}\right)$ is, unlike $p\left(\phi_{n, 0: k}, \epsilon \mid r_{n, 0: k}\right)$, analytically tractable and is obtained via a Kalman filter. Moreover, the marginal posterior distribution $p\left(\phi_{n, 0: k}, \epsilon\right.$ | $\left.r_{n, 0: k}\right)$ can be approximated with a particle filter:

$$
\hat{p}\left(\phi_{n, 0: k}, \epsilon \mid r_{n, 0: k}\right)=\sum_{j=1}^{N} \delta\left(\phi_{n, 0: k}-\phi_{n, 0: k}^{(j)} ; \epsilon-\epsilon^{(j)}\right) \widetilde{w}_{n, k}^{(j)}
$$

where $\delta(\cdot ; \cdot)$ is the two-dimensional Dirac delta function. Thus substituting (25) in (24), we obtain an estimate of the joint a posteriori p.d.f.:

$$
\begin{aligned}
& \hat{p}\left(\phi_{n, 0: k}, \epsilon, \mathbf{s}_{n, k} \mid r_{n, 0: k}\right) \\
& \quad=\sum_{j=1}^{N} p\left(\mathbf{s}_{n, k} \mid \phi_{n, 0: k}^{(j)}, r_{n, 0: k}\right) \delta\left(\phi_{n, 0: k}-\phi_{n, 0: k}^{(j)} ; \epsilon-\epsilon^{(j)}\right) \widetilde{w}_{n, k}^{(j)},
\end{aligned}
$$

where $p\left(\mathbf{s}_{n, k} \mid \phi_{n, 0: k}^{(j)}, r_{n, 0: k}\right)$ is a multivariate Gaussian probability density function with mean $\mathbf{s}_{n, k \mid k}^{(j)}$ and covariance $\Sigma_{n, k \mid k}^{(j)} \cdot \mathbf{s}_{n, k \mid k}^{(j)}$ and $\Sigma_{n, k \mid k}^{(j)}$ are obtained using the kalman filtering equations given by

Time update equations

$$
\left\{\begin{array}{l}
\mathbf{s}_{n, k \mid k-1}^{(j)}=\mathbf{A}_{n, k} \mathbf{s}_{n, k-1 \mid k-1}^{(j)}, \\
\boldsymbol{\Sigma}_{n, k \mid k-1}^{(j)}=\mathbf{A}_{n, k} \boldsymbol{\Sigma}_{n, k-1 \mid k-1}^{(j)} \mathbf{A}_{n, k}^{H}+E\left[\mathbf{b}_{n, k} \mathbf{b}_{n, k}^{H}\right]
\end{array}\right.
$$

Measurement update equations

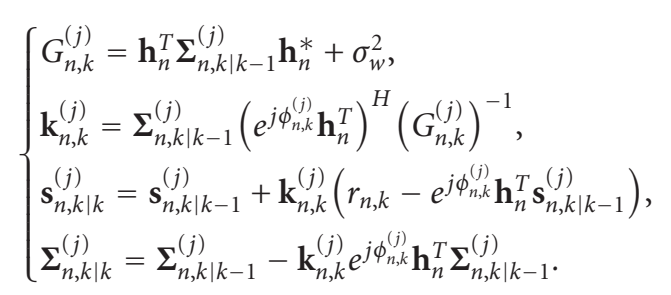

In (28), we can notice that $G_{n, k}^{(j)}$ and $\Sigma_{n, k \mid k}^{(j)}$ are independent of the particle coordinates $\phi_{n, k}^{(j)}$ and thus are identical for all the particles. This remark can be used to reduce the complexity of our algorithm.

Now, the posterior distribution of the multicarrier signal $\mathbf{s}_{n, k}$ is identified, so the remaining task is the simulation of the particles in (26). The marginal posterior distribution can be decomposed as follows:

$$
\begin{aligned}
p\left(\phi_{n, 0: k}, \epsilon \mid r_{n, 0: k}\right)= & C p\left(\phi_{n, 0: k-1} \mid r_{n, 0: k-1}\right) \\
& \times p\left(\epsilon \mid \phi_{n, 0: k-1}, r_{n, 0: k-1}\right) \\
& \times p\left(\phi_{n, k} \mid \phi_{n, 0: k-1}, \epsilon, r_{n, 0: k}\right) \\
& \times p\left(r_{n, k} \mid \phi_{n, 0: k-1}, \epsilon, r_{n, 0: k-1}\right),
\end{aligned}
$$

where $C=\left[p\left(r_{n, k} \mid r_{n, 0: k-1}\right)\right]^{-1}$ is a constant independent on $\phi_{n, 0: k}$ and $\epsilon$, so particle simulation is achieved in four steps. First, $\phi_{n, 0: k-1}$ is simulated from $\hat{p}\left(\phi_{n, 0: k-1} \mid r_{n, 0: k-1}\right)$ obtained at the previous iteration, then $\epsilon$ is simulated from $p\left(\epsilon \mid \phi_{n, 0: k-1}, r_{n, 0: k-1}\right)$ and $\phi_{n, k}$ from $p\left(\phi_{n, k} \mid \phi_{n, k-1}, \epsilon, r_{n, 0: k}\right)$. Finally, particles are accepted with a probability proportional to $p\left(r_{n, k} \mid \phi_{n, 0: k-1}, \epsilon, r_{n, 0: k-1}\right)$. A similar decomposition is used by Storvik [31].

\section{(1) CFO sampling}

At step $k$, the CFO $\epsilon$ is sampled from $p\left(\epsilon \mid \phi_{n, 0: k-1}, r_{n, 0: k-1}\right)$ with

$$
\begin{aligned}
p(\epsilon \mid & \left.\phi_{n, 0: k-1}, r_{n, 0: k-1}\right) \\
& =\frac{p\left(r_{n, 0: k-1} \mid \phi_{n, 0: k-1}, \epsilon\right) p\left(\phi_{n, 0: k-1} \mid \epsilon\right) p(\epsilon)}{p\left(r_{n, 0: k-1}, \phi_{n, 0: k-1}\right)} \\
& \propto p\left(\phi_{n, 0: k-1} \mid \epsilon\right) p(\epsilon) .
\end{aligned}
$$

Firstly, $p\left(\phi_{n, 0: k-1} \mid \epsilon\right)$ is obtained from (4):

$$
\begin{aligned}
& p\left(\phi_{n, 0: k-1} \mid \epsilon\right) \\
& \quad=\mathcal{N}\left(\phi_{n, 0} ; \phi_{n,-1}, \sigma_{v}^{2}\right) \prod_{i=1}^{k-1} \mathcal{N}\left(\phi_{n, i} ; \phi_{n, i-1}+\frac{2 \pi \epsilon}{N}, \sigma_{v}^{2}\right) .
\end{aligned}
$$

Secondly, the CFO $\epsilon$ is assumed to be a uniform random variable on the support $\left[-(\Delta f T)_{\max } ;(\Delta f T)_{\max }\right]$. Consequently, the sampling distribution of $\epsilon$ in (30) can be written as

$$
\begin{aligned}
& p\left(\epsilon \mid \phi_{n, 0: k-1}, r_{n, 0: k-1}\right) \\
& \propto \mathcal{N}\left(\epsilon ; \frac{N \sum_{i=1}^{k-1} \phi_{n, i}-\phi_{n, i-1}}{2 \pi(k-1)}, \frac{\sigma_{v}^{2}}{k-1}\right) \\
& \quad \times U_{\left[-(\Delta f T)_{\max } ;(\Delta f T)_{\max }\right]}(\epsilon),
\end{aligned}
$$

where $U_{[a ; b]}(\epsilon)$ is the uniform distribution on the support $[a ; b]$. This sampling distribution can be viewed as a truncated normal distribution.

\section{(2) Phase distortion sampling}

The choice of the importance function is essential because it determines the efficiency as well as the complexity of the particle filtering algorithm. In this paper, we consider the optimal importance function for $\phi_{n, k}$ which minimizes 
the variance of the importance weights conditional upon the particle trajectories and the observations [32]. In our context, it is expressed as

$$
\pi\left(\phi_{n, k} \mid \phi_{n, 0: k-1}^{(j)}, \epsilon^{(j)}, r_{0: k}\right)=p\left(\phi_{n, k} \mid \phi_{n, 0: k-1}^{(j)}, \epsilon^{(j)}, r_{0: k}\right) .
$$

However, this p.d.f. is analytically intractable. To derive an approximate optimal importance function, it is possible to use the common linearization of the total PHN term $e^{j \theta_{n, k}}=$ $1+j \theta_{n, k}$. However, we propose to linearize only the state update noise, that is, $e^{j v_{n, k}}=1+j v_{n, k}$, where $v_{n, k}$ is defined in (3) which leads to a more accurate approximation. As detailed in Appendix B, this p.d.f. can thus be approximated by

$$
p\left(\phi_{n, k} \mid \phi_{n, 0: k-1}^{(j)}, \epsilon^{(j)}, r_{0: k}\right) \approx \mathcal{N}\left(\phi_{n, k} ; \mu_{n, k}^{(j)}, \Lambda_{k}^{(j)}\right)
$$

where

$$
\begin{aligned}
& \mu_{n, k}^{(j)}= \begin{cases}\gamma_{0}^{(j)}, & \text { if } k=0 \\
\gamma_{k}^{(j)}+\phi_{n, k-1}^{(j)}+\frac{2 \pi \epsilon^{(j)}}{N}, & \text { otherwise, }\end{cases} \\
& \Lambda_{k}^{(j)}=\frac{\chi_{k}^{(j)} \sigma_{v}^{2}}{\left|\Gamma_{k}^{(j)}\right|^{2} \sigma_{v}^{2}+\chi_{k}^{(j)}}
\end{aligned}
$$

with $\gamma_{k}^{(j)}=\mathfrak{I}\left(\Gamma_{k}^{(j) *} r_{n, k}\right) \sigma_{v}^{2} /\left(\left|\Gamma_{k}^{(j)}\right|^{2} \sigma_{v}^{2}+\chi_{k}^{(j)}\right)$, (where $\mathfrak{I}(\cdot)$ denotes the imaginary part), $\chi_{k}^{(j)}=\mathbf{h}_{n}^{T} \boldsymbol{\Sigma}_{n, k \mid k-1}^{(j)} \mathbf{h}_{n}^{*}+\sigma_{b}^{2}$ and

$$
\Gamma_{k}^{(j)}= \begin{cases}\mathbf{h}_{n}^{T} \mathbf{s}_{n, 0 \mid-1}^{(j)}, & \text { if } k=0 \\ e^{j\left(\phi_{n, k-1}^{(j)}+2 \pi \epsilon^{(j)} / N\right)} \mathbf{h}_{n}^{T} \mathbf{s}_{n, k \mid k-1}^{(j)}, & \text { otherwise, }\end{cases}
$$

where $\mathbf{s}_{n, 0 \mid-1}^{(j)}$ is only composed of the $L-1$ signal estimate samples obtained for the previous $n-1$ th multicarrier symbol.

\section{(3) Evaluation of the importance weights}

Using (21), the importance weights in the proposed marginalized particle filter are updated according to the relation:

$$
w_{n, k}^{(j)} \propto w_{n, k-1}^{(j)} \frac{p\left(r_{n, k} \mid \phi_{n, k}^{(j)}, r_{n, k-1}\right) p\left(\phi_{n, k}^{(j)} \mid \phi_{n, k-1}^{(j)}, \epsilon^{(j)}\right)}{\hat{p}\left(\phi_{n, k}^{(j)} \mid \phi_{n, 0: k-1}^{(j)}, \epsilon^{(j)}, r_{n, 0: k}\right)},
$$

where $\hat{p}\left(\phi_{n, k}^{(j)} \mid \phi_{n, 0: k-1}^{(j)}, \epsilon^{(j)}, r_{n, 0: k}\right)$ is the approximate optimal importance function given by (34),

$$
p\left(r_{n, k} \mid \phi_{n, k}^{(j)}, r_{n, k-1}\right)=\mathcal{N}_{c}\left(r_{n, k} ; e^{j \phi_{n, k}^{(j)}} \mathbf{h}_{n}^{T} \mathbf{s}_{n, k \mid k-1}^{(j)}, \mathbf{G}_{n, k}^{(j)}\right)
$$

with $\mathbf{G}_{n, k}^{(j)}$ the innovation covariance of the $j$ th kalman filter given by (28) and the prior distribution of $\phi_{n, k}$ is given using (4) by

$$
\begin{aligned}
& p\left(\phi_{n, k}^{(j)} \mid \phi_{n, k-1}^{(j)}, \epsilon^{(j)}\right) \\
& = \begin{cases}\mathcal{N}\left(\phi_{n, 0}^{(j)} ; 0, \sigma_{v}^{2}\right), & \text { if } k=0 \\
\mathcal{N}\left(\phi_{n, i}^{(j)} ; \phi_{n, i-1}^{(j)}+\frac{2 \pi \epsilon^{(j)}}{N}, \sigma_{v}^{2}\right) & \text { otherwise. }\end{cases}
\end{aligned}
$$

\section{(4) MMSE estimate of multicarrier signal, PHN and CFO}

Every element required in the implementation of the marginalized particle filtering algorithm has been identified. The resulting weighted samples $\left\{\mathbf{s}_{n, k \mid k}^{(j)}, \Sigma_{n, k \mid k}^{(j)}, \phi_{n, 0: k}^{(j)}\right.$, $\left.\epsilon^{(j)}, \widetilde{w}_{n, k}^{(j)}\right\}_{j=1}^{M}$ approximate the posterior density function $p\left(\mathbf{s}_{n, k}, \phi_{n, 0: k}, \epsilon \mid r_{n, 0: k}\right)$. Consequently, the minimum mean square error (MMSE) estimates of $\mathbf{s}_{n, k}, \phi_{n, k}$, and $\epsilon$ are obtained at the end of the $n$th OFDM symbol by the respective expressions:

$$
\begin{gathered}
\widehat{\mathbf{s}}_{n, N+N_{\mathrm{cp}}-1}=\sum_{j=1}^{N} \widetilde{w}_{n, N+N_{\mathrm{cp}}-1}^{(j)} \mathbf{s}_{n, N+N_{\mathrm{cp}}-1 \mid N+N_{\mathrm{cp}}-1}^{(j)}, \\
\hat{\phi}_{n, 0: N+N_{\mathrm{cp}}-1}=\sum_{j=1}^{N} \widetilde{w}_{n, N+N_{\mathrm{cp}}-1}^{(j)} \phi_{n, 0: N+N_{\mathrm{cp}}-1}^{(j)}, \\
\hat{\epsilon}=\sum_{j=1}^{N} \widetilde{w}_{n, N+N_{\mathrm{cp}}-1}^{(j)} \epsilon^{(j)} .
\end{gathered}
$$

The proposed marginalized particle filter algorithm, denoted by JSCPE-MPF where this acronym stands for joint signal, CFO, and PHN estimation using marginalized particle filter, is summed up in Algorithm 1.

\subsection{The posterior Cramér-Rao bound}

In order to study the efficiency of an estimation method, it is of great interest to compute the variance bounds on the estimation errors and to compare them to the lowest bounds corresponding to the optimal estimator. For timeinvariant statistical models, a commonly used lower bound is the Cramér-Rao bound (CRB), given by the inverse of the Fisher information matrix. In a time-varying context as we deal with here, a lower bound analogous to the CRB for random parameters has been derived in [33]; this bound is usually referred to as the Van Trees version of the CRB, or posterior CRB (PCRB) [34].

Unfortunately, the PCRB for the joint estimation of $\left\{\phi_{n, k}, \epsilon, \mathbf{s}_{n, k}\right\}$ is analytically intractable. Since the multicarrier signal is the main quantity of interest, we derive in this paper the conditional PCRB of $\mathbf{s}_{n, k}$, where $\left\{\phi_{n, k}, \epsilon\right\}$ are assumed perfectly known. Under this assumption, the DSS model (19) becomes linear and Gaussian and the PCRB obtained at the end of each OFDM symbol is equal to the covariance 


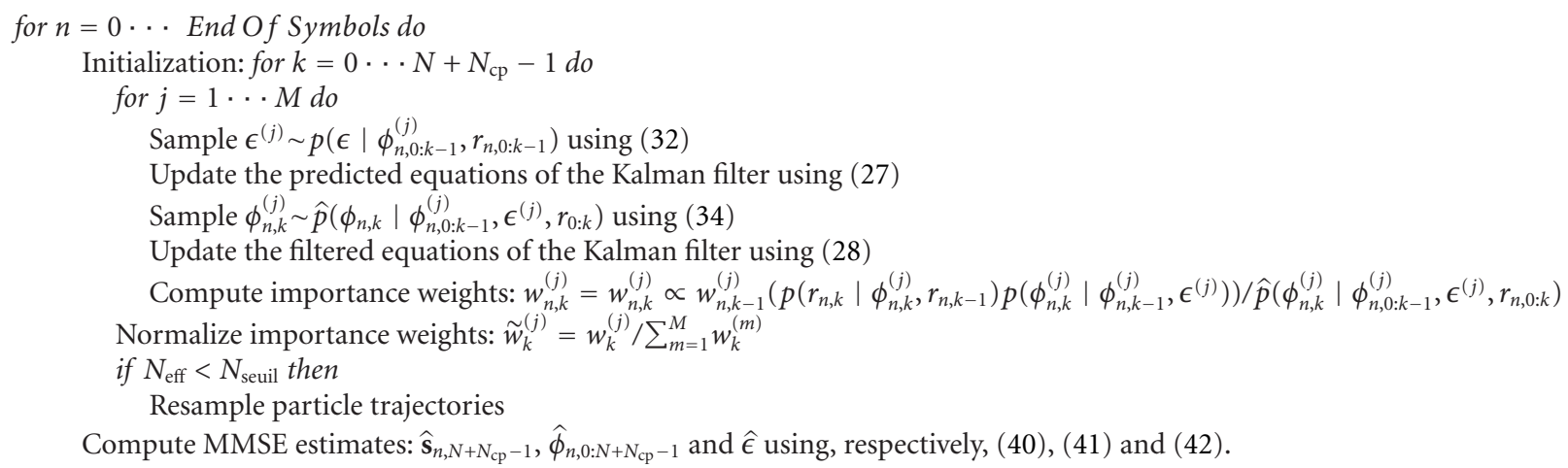

Algorithm 1: JSCPE-MPF algorithm.

matrix $\boldsymbol{\Sigma}_{n, N+N_{\mathrm{cp}}-1 \mid N+N_{\mathrm{cp}}-1}$ of the posterior p.d.f. $p\left(\mathbf{s}_{n, N+N_{\mathrm{cp}}-1} \mid\right.$ $\left.\phi_{n, 0: N+N_{\mathrm{cp}}-1}, \epsilon, r_{n, 0: N+N_{\mathrm{cp}}-1}\right)$ given by the kalman filter [35]:

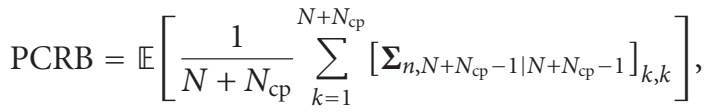

where $\left[\boldsymbol{\Sigma}_{n, N+N_{\text {cp }}-1 \mid N+N_{\text {cp }}-1}\right]_{k, k}$ denotes the $(k, k)$ th entry of the matrix $\boldsymbol{\Sigma}_{n, N+N_{\text {cp }}-1 \mid N+N_{\text {cp }}-1}$. This PCRB is estimated using the Monte Carlo method by recursively evaluating the predicted and filtered equations (27), (28), where $\left\{\phi_{n, k}, \epsilon\right\}$ are set to their true values.

\section{RESULTS}

In order to show the validity of our approach, extensive simulations have been performed. In a first part, the case of PHN without CFO is considered. The performances of the proposed JSCPE-MPF are then compared to the nonpilot-aided variational scheme proposed in [17] and to a CPE correction with a perfect knowledge of the CPE value corresponding to the ideal case of [16]. In the last part, the performances of the JSCPE-MPF are assessed when both $\mathrm{CFO}$ and $\mathrm{PHN}$ are present in multicarrier systems.

In all these cases, performances are shown in terms of mean square error (MSE) and bit error rate (BER). Since the joint estimation is carried out in the time domain with the JSCPE-MPF, the MSE performance of the JSCPE-MPF estimates holds whatever the multicarrier system used. For comparison purposes, the BER performance of a multicarrier system using a frequency domain MMSE equalizer (denoted by MMSE-FEQ) without phase distortions is also depicted.

With regard to the system parameters, 16-QAM modulation is assumed and we have chosen $N=64$ subcarriers with a cyclic prefix of length $N_{\text {cp }}=8$. A Rayleigh frequency selective channel with $L=4$ paths and a uniform power delay profile, perfectly known by the receiver, has been generated for each OFDM symbol. The proposed JSCPE-MPF has been implemented with 100 particles.

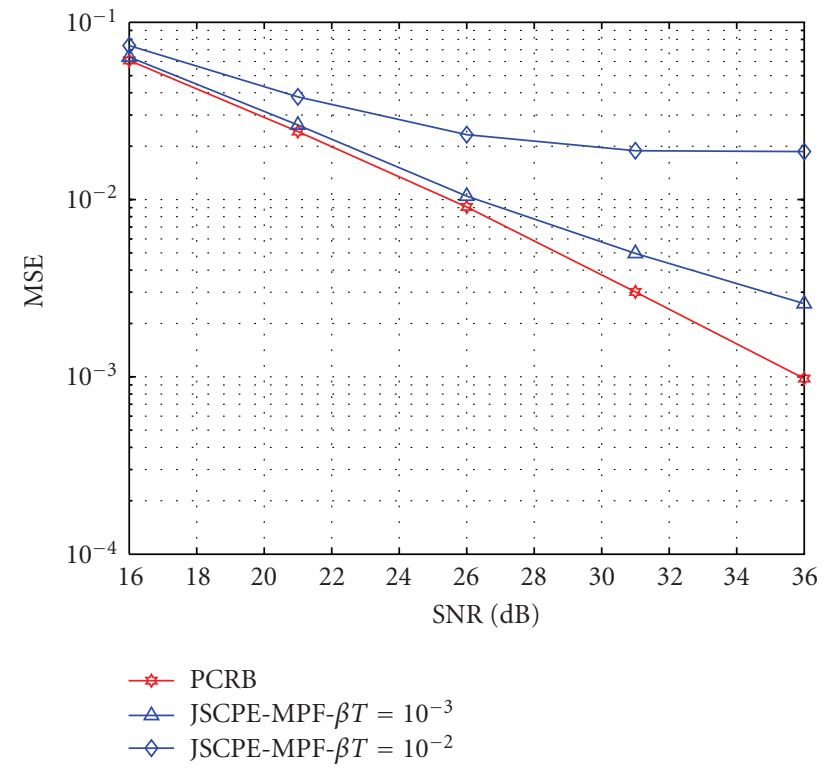

FIGURE 4: MSE of the multicarrier signal estimate versus SNR for different PHN rates $\beta T(\epsilon=0)$.

\subsection{Performances with PHN only (i.e., $\epsilon=0$ )}

We first perform simulations with no $\mathrm{CFO}$ in order to study the joint PHN and multicarrier signal estimation. The multicarrier signal estimation performance of JSCPE-MPF is shown in Figure 4. The performance of the proposed estimator is compared to the posterior Cramér-Rao bound (PCRB) of a multicarrier system without phase distortions derived in Section 4.3. For a small phase noise rate $\beta T$, it can be seen that the proposed JSCPE-MPF almost achieves the optimal performance without PHN given by the PCRB. Consequently, the proposed approximate optimal importance function for the PHN sampling yields an efficient non-pilotbased algorithm.

Figure 5 depicts the BER performance of the JSCPE-MPF algorithm compared to the variational scheme proposed in 


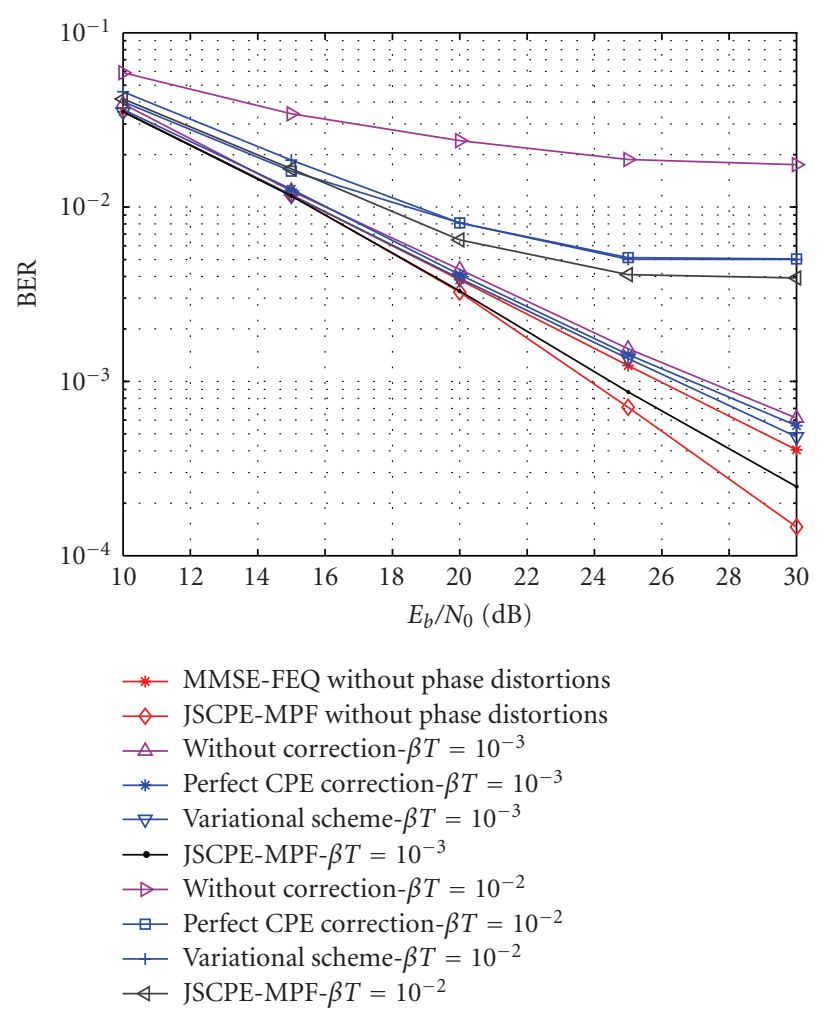

FIGURE 5: BER performance of the proposed JSCPE-MPF versus $E_{b} / N_{0}$ for different PHN rates $\beta T$ in an OFDM system $(\epsilon=0)$.

[17] and to a perfect CPE correction scheme. Since the multicarrier signal estimation is achieved by a Kalman filter, we can denote that, in a phase distortion-free context and by excluding the cyclic prefix in the received signal, the proposed algorithm leads to a time-domain MMSE equalizer. Time-domain and frequency-domain MMSE equalization are mathematically equivalent and result in the same performance [36]. Consequently, the performance gain between the MMSE-FEQ and the JSCPE-MPF without distortions clearly highlights the benefit of considering the additional information induced by the cyclic prefix. As depicted in Figure 5, the proposed JSCPE-MPF outperforms conventional schemes whatever the PHN rate. Moreover, for $\beta T=10^{-3}$, the JSCPE-MPF curve is close to the optimal bound and outperforms the MMSE-FEQ without PHN.

\subsection{Performances with both PHN and CFO}

In the following simulations, the $\mathrm{CFO}$ term $\epsilon$ is generated from a uniform distribution in $[-0.5 ; 0.5]$, that is, half of the subcarrier spacing. The performance of the JSCPEMPF joint estimation is first studied in term of the mean square error (MSE). First, we focus on the phase distortion estimation performance. Figure 6 shows the corresponding MSE is plotted versus the signal-to-noise ratio (SNR). Even with significant PHN rate and large CFO, JSCPEMPF achieves accurate estimation of the phase distortions. The MSE curves tend toward a minimum MSE threshold depending on PHN rate $\beta T$. Figure 7 depicts the multicarrier

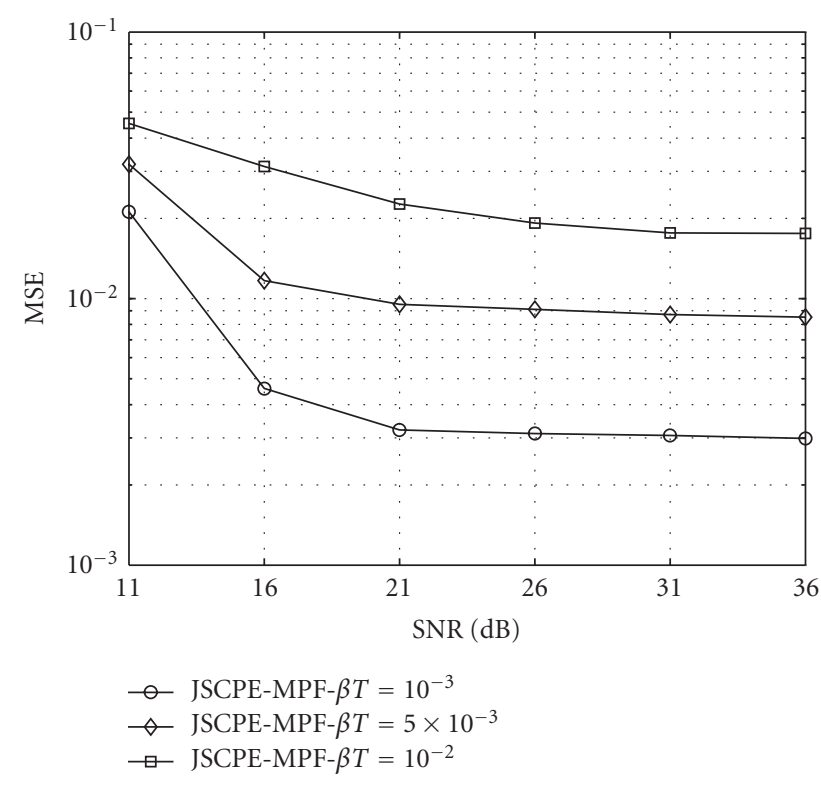

FIGURE 6: MSE of phase distortion estimate versus SNR for different PHN rates $\beta$ T.

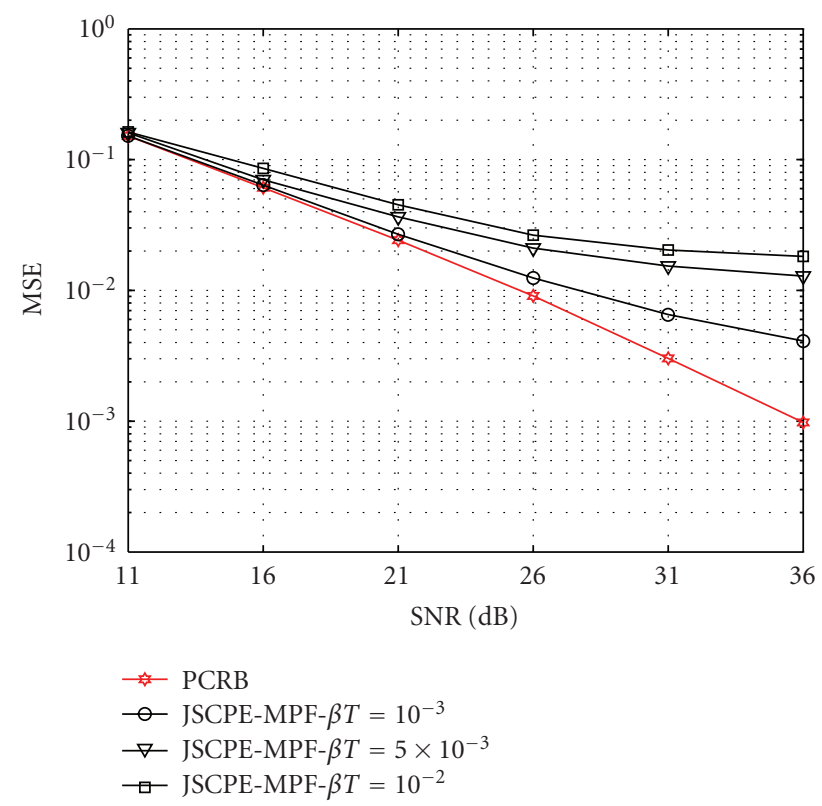

FIGURE 7: MSE of the multicarrier signal estimate versus SNR for different $\mathrm{PHN}$ rates $\beta T$.

signal estimation accuracy as a function of the SNR. The performance of the proposed estimator is compared to the PCRB. For $\beta T=10^{-3}$, it performs close to the PCRB. The gap between the PCRB and the JSCPE-MPF increases with $\mathrm{PHN}$ rate since the phase distortions get stronger. Moreover, by comparing MSE results depicted in Figure 4, where phase distortions are reduced to $\mathrm{PHN}$, it can be denoted that the signal estimation performance is slightly degraded when 


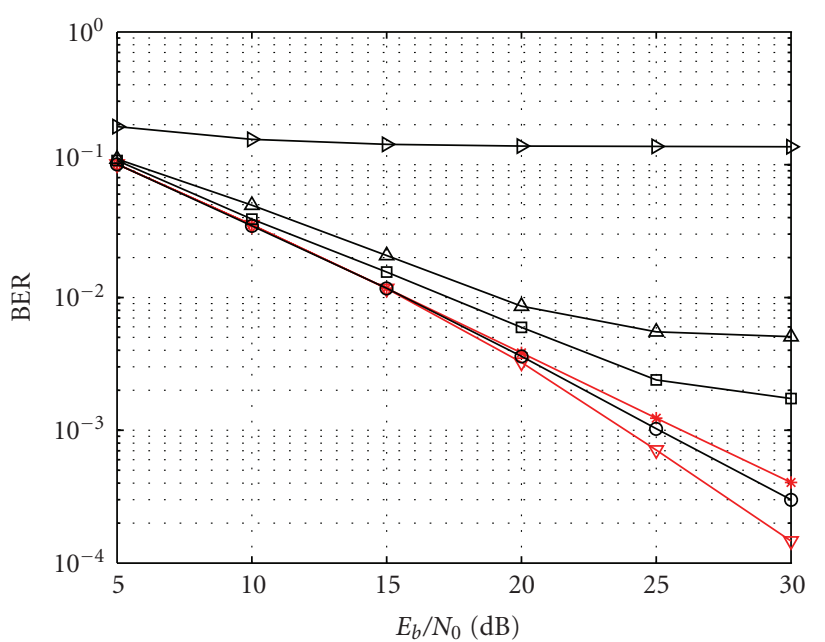

$$
\begin{aligned}
& * \text { MMSE-FEQ without phase distortions } \\
& \nabla \text { JSCPE-MPF without phase distortions } \\
& - \text { JSCPE-MPF- } \beta T=10^{-3} \\
& \square \text { JSCPE-MPF- } \beta T=5 \times 10^{-3} \\
& \triangle \text { JSCPE-MPF- } \beta T=10^{-2} \\
& \square \text { Perfect CPE correction- } \beta T=10^{-3}
\end{aligned}
$$

FIGURE 8: BER performance of the proposed JSCPE-MPF versus $E_{b} / N_{0}$ with severe CFO and for different PHN rates $\beta T$ in an OFDM system.

considering CFO. These MSE results illustrate the accuracy of both the CFO and the PHN sampling strategy.

Now, we study the BER performance of the proposed JSCPE-MPF for two different multicarrier systems: the OFDM and the MC-CDMA systems. Figure 8 depicts the BER performance as a function of signal-to-noise ratio for different PHN rates in an OFDM system. First, for the perfect $\mathrm{CPE}$ correction scheme, an error floor exists because of the residual ICI. In this case, it is obvious that any decisiondirected-based algorithms lead to poor performance. The importance of considering the redundancy information given by the cyclic prefix is illustrated by the gain between the MMSE-FEQ and the JSCPE-MPF without phase distortions. For $\beta T=10^{-3}$, the proposed JSCPE-MPF still outperforms the MMSE-FEQ without phase distortions. Moreover, even if the PHN rate increases, the JSCPE-MPF still achieves accurate estimation.

Finally, the BER performances of the JSCPE-MPF for a full and half-loaded MC-CDMA system are, respectively, shown in Figures 9-10. Since the downlink transmission is time-synchronous, Walsh codes are selected for their orthogonality property. From these figures, we observe that the JSCPE-MPF slightly outperforms the MMSE-FEQ without phase distortions for both a full and a half-loaded system. This is principally due to the cyclic prefix additional information. Moreover, since the estimation accuracy of the proposed algorithm does not depend on the system load (Figure 7), the performance gap between a full and a half-loaded system is simply explained by the multipleaccess interference (MAI) induced by the frequency selective channel at the data detection stage.

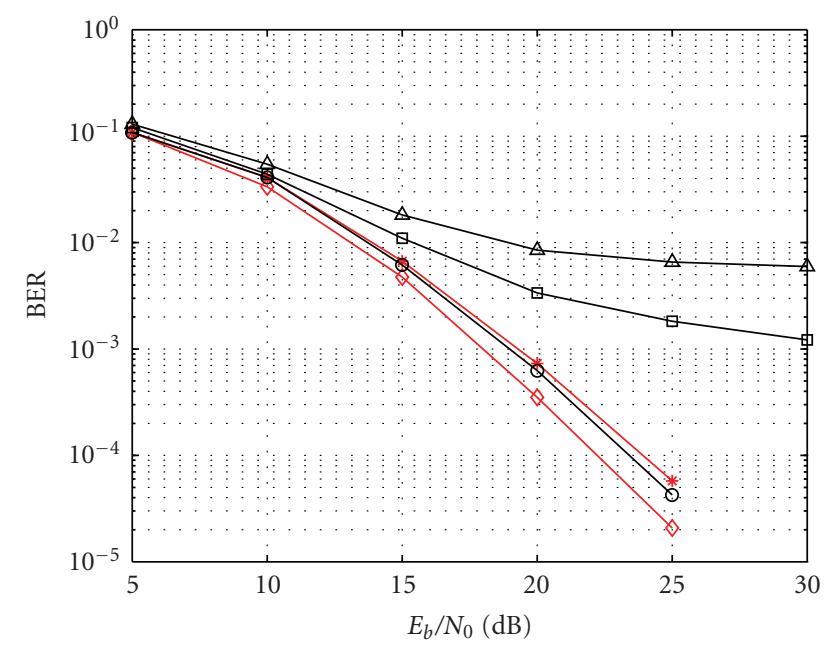

$$
\begin{aligned}
& \rightarrow \text { MMSE-FEQ without phase distortions } \\
& \diamond \text { JSCPE-MPF without phase distortions } \\
& - \text { JSCPE-MPF- } \beta T=10^{-3} \\
& \square \text { JSCPE-MPF- } \beta T=5 \times 10^{-3} \\
& \triangle \text { JSCPE-MPF- } \beta T=10^{-2}
\end{aligned}
$$

FIGURE 9: BER performance of the proposed JSCPE-MPF versus $E_{b} / N_{0}$ for different PHN rates $\beta T$ in a full-loaded MC-CDMA system.

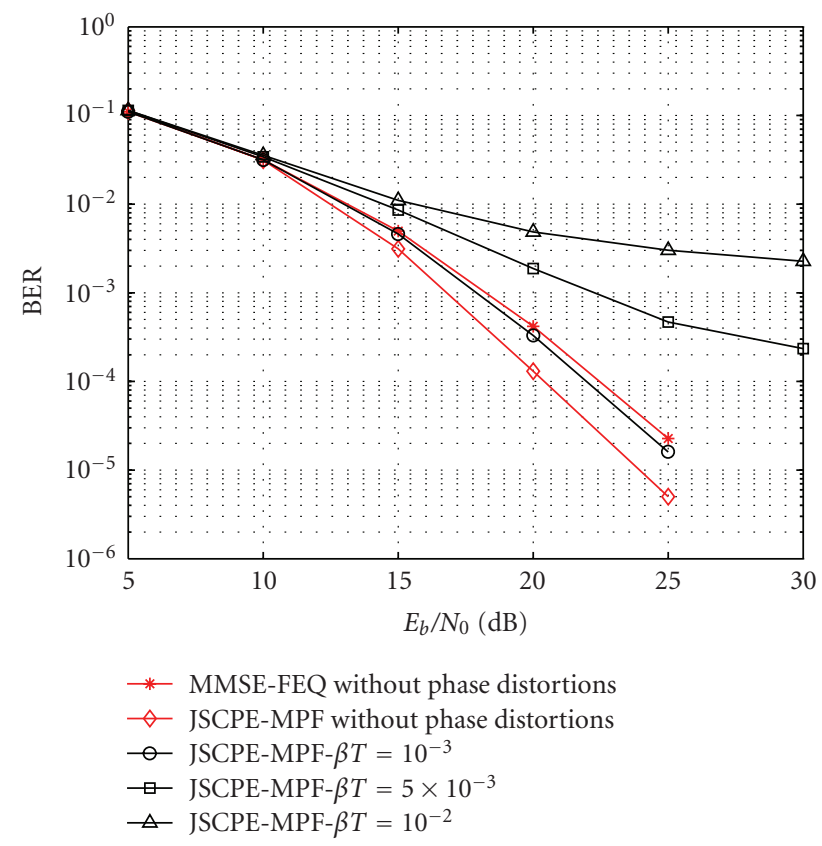

FIGURE 10: BER performance of the proposed JSCPE-MPF versus $E_{b} / N_{0}$ for different PHN rates $\beta T$ in a half-loaded MC-CDMA system.

\section{CONCLUSION}

The paper deals with the major problem of multicarrier systems that suffer from the presence of phase noise (PHN) and carrier frequency offset (CFO). The originality of this work consists in using the sequential Monte Carlo methods 
justified by the nonlinear behavior of the received signal. Moreover, a time processing strategy is proposed in order to take into account the redundancy information induced by the cyclic prefix and to accurately estimate the time variations of the phase distortions. Finally, the proposed scheme has the advantage of being bandwidth efficient as it does not require the transmission of pilot symbols.

Numerical simulations show that even with significant PHN rates and severe CFO, the JSCPE-MPF achieves good performances both in terms of the phase distortion estimation and BER. In particular, it was found that, for a small PHN rate, the JSCPE-MPF is more efficient in term of BER than a frequency MMSE equalizer in a system without phase distortions. Compared to the variational scheme proposed in [17], the JSCPE-MPF has better performance when only PHN degrades the transmitted signal. Moreover, unlike this algorithm, the JSCPE-MPF enables to cope with severe CFO. According to simulation results, our algorithm also outperforms the algorithm based only on the CPE correction [16] whatever the PHN rate and the CFO. As a consequence, the JSCPE-MPF algorithm offers a significant performance gain in comparison to existing methods and can be efficiently used with the channel estimator proposed in [22] for the design of a complete multicarrier receiver in wireline and wireless communication systems.

\section{APPENDIX}

\section{A. ON THE GAUSSIAN APPROXIMATION OF THE MULTICARRIER SIGNAL}

Let us recall that the transmitted multicarrier signal is given, for $t=0, \ldots, N-1$, by

$$
s_{n, t}=\frac{1}{\sqrt{N}} \sum_{i=0}^{N-1} d_{n, i} e^{j 2 \pi i t / N},
$$

where $\left\{d_{n, i}\right\}_{i=0}^{N-1}$ are a function of user data symbols and spreading codes, so depending of the multicarrier system considered. Since $\left\{d_{n, i}\right\}_{i=0}^{N-1}$ are i.i.d. variables with unitary power and according to the central limit theorem, we can expect that this multicarrier signal can be approximated as a circular Gaussian distributed random variable.

In order to study precisely the accuracy of the Gaussian approximation, Figure 11 presents the maximum gaps between the cumulative distribution function (CDF) of both the real and imaginary part of the OFDM signal, denoted by $F(\cdot)$ and the CDF of a Gaussian random variable with zero mean and variance $1 / 2$, denoted by $G(\cdot)$. This gap is thus defined as $\max _{x}|F(x)-G(x)|$.

For simplicity, an OFDM system is assumed in this appendix and thus $\left\{d_{n, i}\right\}_{i=0}^{N-1}$ are directly the user data symbol. Moreover, 2,000,000 OFDM symbols have been generated for each system configuration.

From these figures, it can be seen logically that the accuracy of a Gaussian approximation for the multicarrier signal increases with both the number of subcarriers and the size of the constellation. Moreover, the approximation accuracy for the real and imaginary part of the multicarrier is identical except for the BPSK case. In fact, this difference with the use of BPSK can be simply explained by (A.1). Indeed, when BPSK is used, both $s_{n, 0}$ and $s_{n, N / 2}$ have no imaginary part, that is,

$$
\begin{aligned}
s_{n, 0} & =\frac{1}{\sqrt{N}} \sum_{i=0}^{N-1} d_{n, i}, \\
s_{n, N / 2} & =\frac{1}{\sqrt{N}} \sum_{i=0}^{N-1} d_{n, i}(-1)^{i} .
\end{aligned}
$$

As a consequence, with BPSK modulation, the Gaussian approximation of the real and imaginary part of the OFDM signal can be summarized as follows:

$$
\begin{aligned}
& \mathfrak{R}\left(s_{n, t}\right) \sim \begin{cases}\mathcal{N}(0,1), & \text { if } t=0, t=\frac{N}{2} \\
\mathcal{N}\left(0, \frac{1}{2}\right), & \text { otherwise }\end{cases} \\
& \mathfrak{I}\left(s_{n, t}\right) \sim \begin{cases}\delta\left(\mathfrak{I}\left(s_{n, t}\right)\right), & \text { if } t=0, t=\frac{N}{2} \\
\mathcal{N}\left(0, \frac{1}{2}\right), & \text { otherwise. }\end{cases}
\end{aligned}
$$

These adaptations have to be taken into account in the proposed multicarrier receiver if the BPSK modulation is used.

From these results, it can be concluded that the Gaussian distribution $\mathcal{N}(0,1 / 2)$ represents an accurate model for both the real and imaginary part of the transmitted multicarrier signal, except in the BPSK case for $t=0$ and $t=N / 2$.

\section{B. DERIVATION OF THE OPTIMAL IMPORTANCE FUNCTION FOR PHN SAMPLING}

The optimal importance function for phase distortion $\phi_{n, k}$ sampling in the proposed particle filter requires the derivation of the p.d.f. defined in (33). This p.d.f. can be rewritten as

$$
\begin{aligned}
& p\left(\phi_{n, k} \mid \phi_{n, 0: k-1}^{(j)}, \epsilon^{(j)}, r_{0: k}\right) \\
= & \frac{p\left(r_{k} \mid \phi_{n, k}, \phi_{n, 0: k-1}^{(j)}, \epsilon^{(j)}, r_{0: k-1}\right) p\left(\phi_{n, k} \mid \phi_{n, 0: k-1}^{(j)}, \epsilon^{(j)}\right)}{\int p\left(r_{k} \mid \phi_{n, k}, \phi_{n, 0: k-1}^{(j)}, \epsilon^{(j)}, r_{0: k-1}\right) p\left(\phi_{n, k} \mid \phi_{n, 0: k-1}^{(j)}, \epsilon^{(j)}\right) d \phi_{n, k}}
\end{aligned}
$$

with

$$
\begin{array}{ll}
p\left(\phi_{n, k} \mid\right. & \left.\phi_{n, 0: k-1}^{(j)}, \epsilon^{(j)}\right) \\
= & \begin{cases}\mathcal{N}\left(\phi_{n, k} ; 0, \sigma_{v}^{2}\right), & \text { if } k=0 \\
\mathcal{N}\left(\phi_{n, k} ; \phi_{n, k-1}^{(j)}+\frac{2 \pi \epsilon^{(j)}}{N}, \sigma_{v}^{2}\right), & \text { otherwise }\end{cases} \\
p\left(r_{n, k} \mid \phi_{n, k}, \phi_{n, 0: k-1}^{(j)}, \epsilon^{(j)}, r_{n, 0: k-1}\right) & \\
= & \left\{\left(r_{n, k} \mid \mathbf{s}_{n, k}, \phi_{n, k}, \phi_{n, 0: k-1}^{(j)}, \epsilon^{(j)}, r_{n, 0: k-1}\right)\right.
\end{array}
$$




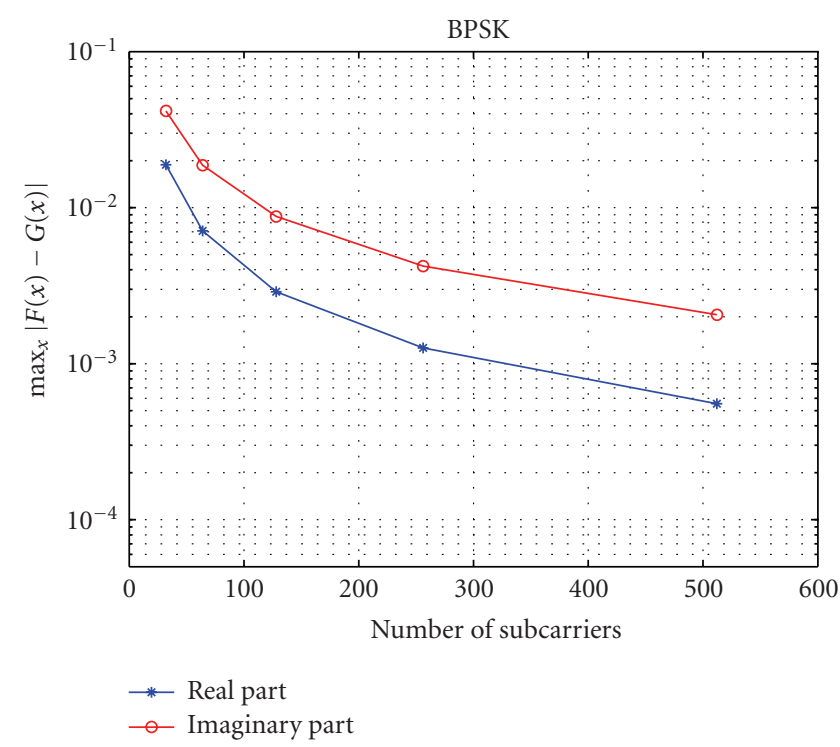

(a)

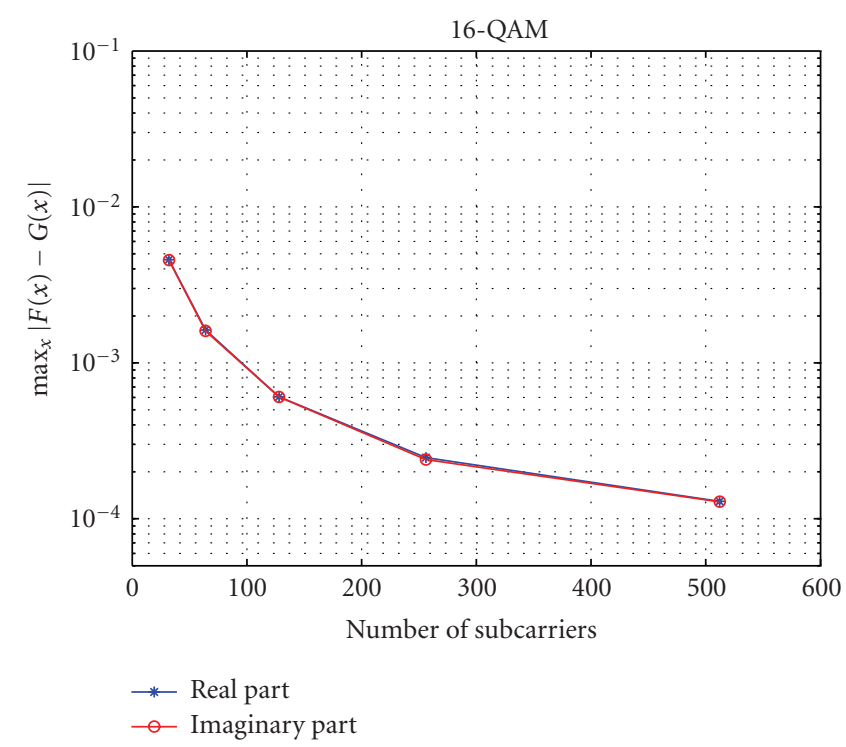

(c)

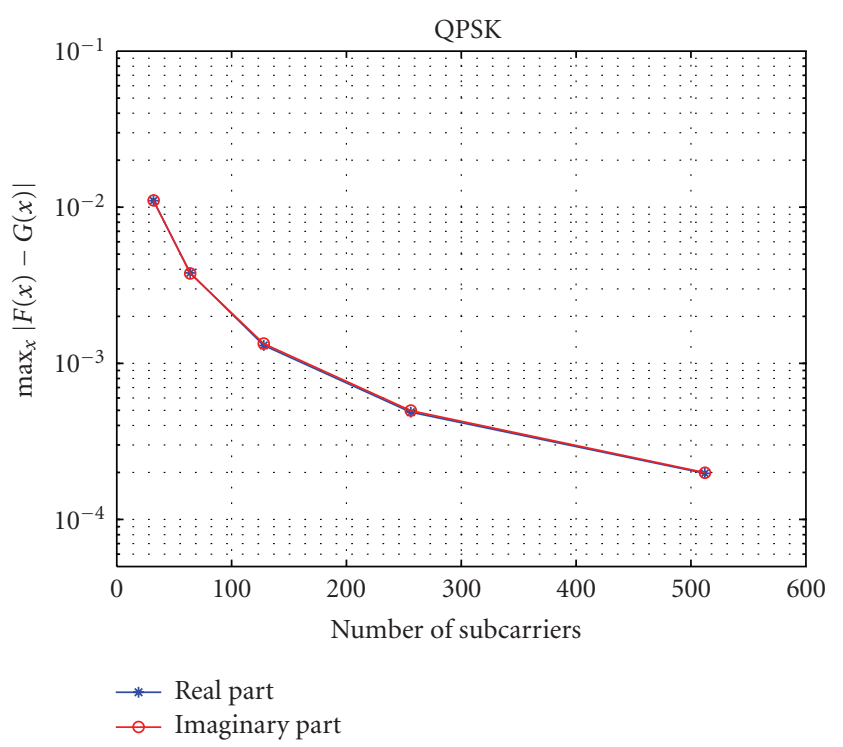

(b)

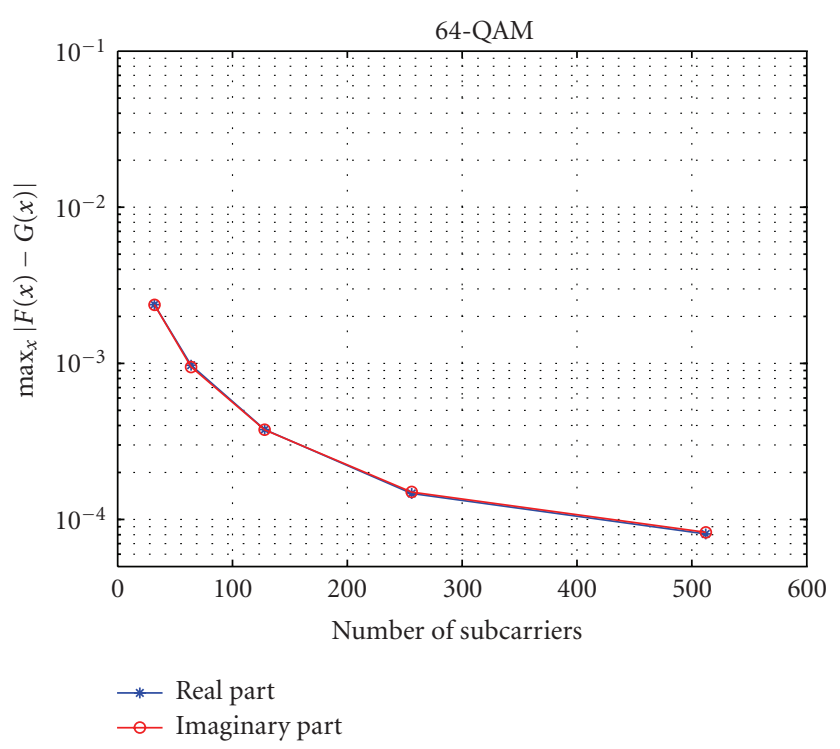

(d)

FIGURE 11: Maximum gaps between the CDF of both the real and imaginary part of the OFDM signal and the CDF of a Gaussian random variable with zero mean and variance $1 / 2$.

The summand in (B.3) is the product of the likelihood p.d.f. and the posterior p.d.f. of the multicarrier signal $\mathbf{s}_{n, k}$ which are respectively given by

$$
\begin{gathered}
p\left(r_{n, k} \mid \mathbf{s}_{n, k}, \phi_{n, k}, \phi_{n, 0: k-1}^{(j)}, \epsilon^{(j)}, r_{0: k-1}\right) \\
=\mathcal{N}_{c}\left(r_{k} ; e^{j \phi_{n, k}} \mathbf{h}_{n}^{T} \mathbf{s}_{n, k}, \sigma_{b}^{2}\right), \\
p\left(\mathbf{s}_{n, k} \mid \phi_{n, 0: k-1}^{(j)}, \epsilon^{(j)}, r_{0: k-1}\right) \\
=\mathcal{N}_{c}\left(\mathbf{s}_{n, k} ; \mathbf{s}_{n, k-1 \mid k-1}, \mathbf{\Sigma}_{n, k-1 \mid k-1}^{(j)}\right) .
\end{gathered}
$$

Therefore, using (B.4), it is straightforward to show that the expression described in (B.3) can be rewritten as

$$
p\left(r_{n, k} \mid \phi_{n, k}, \phi_{n, 0: k-1}^{(j)}, \epsilon^{(j)}, r_{n, 0: k-1}\right)=\mathcal{N}_{c}\left(r_{n, k} ; \rho_{k}^{(j)}, \chi_{k}^{(j)}\right),
$$

where $\rho_{k}^{(j)}=e^{j \phi_{n, k}^{(j)}} \mathbf{h}_{n}^{T} \mathbf{s}_{n, k \mid k-1}^{(j)}$ and $\chi_{k}^{(j)}=\mathbf{h}_{n}^{T} \Sigma_{n, k \mid k-1}^{(j)} \mathbf{h}_{n}^{*}+$ $\sigma_{b}^{2}$. According to (B.2)-(B.5), an analytical form for (B.1) remains untractable due to the double exponential in (B.5). 
However, by linearizing $e^{j v_{n, k}}$, where the noise term $v_{n, k}$ is defined by (4), the mean of (B.5) is approximated by

$$
\rho_{k}^{(j)} \approx \begin{cases}\left(1+j v_{n, 0}\right) \mathbf{h}_{n}^{T} \mathbf{s}_{n, 0 \mid-1}^{(j)}, & \text { if } k=0 \\ \left(1+j v_{n, k}\right) e^{j\left(\phi_{n, k-1}^{(j)}+2 \pi \epsilon^{(j)} / N\right)} \mathbf{h}_{n}^{T} \mathbf{s}_{n, k \mid k-1}^{(j)} & \text { otherwise. }\end{cases}
$$

This approximation holds when the phase distortion power is small and is more accurate than the usual approximation $e^{j \phi_{n, k}} \approx 1+j \phi_{n, k}$. Using (B.2), (B.5), and (B.6) and after several algebraic manipulations, the numerator of (B.1) can be simplified as

$$
\begin{aligned}
p\left(r_{k} \mid\right. & \left.\phi_{n, k}, \phi_{n, 0: k-1}^{(j)}, \epsilon^{(j)}, r_{0: k-1}\right) p\left(\phi_{n, k} \mid \phi_{n, 0: k-1}^{(j)}, \epsilon^{(j)}\right) \\
\approx & \Upsilon_{k}^{(j)} e^{-\left(1 / 2 \Lambda_{k}^{(j)}\right)\left[\left|r_{n, k}-\Gamma_{k}^{(j)}\right|^{2} /\left(\left|\Gamma_{k}^{(j)}\right|^{2} \sigma_{v}^{2}+\chi_{k}^{(j)}\right)-\left|\gamma_{k}^{(j)}\right|^{2}\right]} \\
& \times \mathcal{N}\left(\phi_{n, k} ; \mu_{n, k}^{(j)}, \Lambda_{k}^{(j)}\right),
\end{aligned}
$$

where

$$
\mu_{n, k}^{(j)}= \begin{cases}\gamma_{0}^{(j)}, & \text { if } k=0 \\ \gamma_{k}^{(j)}+\phi_{n, k-1}^{(j)}+\frac{2 \pi \epsilon^{(j)}}{N}, & \text { otherwise }\end{cases}
$$

and $\Upsilon_{k}^{(j)}=\sqrt{\Lambda_{k}^{(j)} /\left(2 \pi \chi_{k}^{(j)} \sigma_{v}^{2}\right)}, \gamma_{k}^{(j)}=\Im\left(\Gamma_{k}^{(j) *} r_{n, k}\right) \sigma_{v}^{2} /\left(\left|\Gamma_{k}^{(j)}\right|^{2} \sigma_{v}^{2}+\right.$ $\left.\chi_{k}^{(j)}\right)$ (with $\mathfrak{I}(\cdot)$ the imaginary part), $\Lambda_{k}^{(j)}=\chi_{k}^{(j)} \sigma_{v}^{2} /\left(\left|\Gamma_{k}^{(j)}\right|^{2} \sigma_{v}^{2}+\right.$ $\left.\chi_{k}^{(j)}\right)$ and

$$
\Gamma_{k}^{(j)}= \begin{cases}\mathbf{h}_{n}^{T} \mathbf{s}_{n, 0 \mid-1}^{(j)}, & \text { if } k=0 \\ e^{j\left(\phi_{n, k-1}^{(j)}+2 \pi \epsilon^{(j)} / N\right)} \mathbf{h}_{n}^{T} \mathbf{s}_{n, k \mid k-1}^{(j)} & \text { otherwise. }\end{cases}
$$

Therefore, from (B.7), it is obvious that

$$
\begin{gathered}
\int p\left(r_{k} \mid \phi_{n, k}, \phi_{n, 0: k-1}^{(j)}, \epsilon^{(j)}, r_{0: k-1}\right) p\left(\phi_{n, k} \mid \phi_{n, 0: k-1}^{(j)}, \epsilon^{(j)}\right) d \phi_{n, k} \\
\approx \Upsilon_{k}^{(j)} e^{-\left(1 / 2 \Lambda_{k}^{(j)}\right)\left[\left|r_{n, k}-\Gamma_{k}^{(j)}\right|^{2} /\left(\left|\Gamma_{k}^{(j)}\right|^{2} \sigma_{v}^{2}+\chi_{k}^{(j)}\right)-\left|\gamma_{k}^{(j)}\right|^{2}\right]} .
\end{gathered}
$$

Finally, the optimal importance sampling for $\phi_{n, k}$ defined in (B.1) can be approximated by

$$
p\left(\phi_{n, k} \mid \phi_{n, 0: k-1}^{(j)}, \epsilon^{(j)}, r_{0: k}\right) \approx \mathcal{N}\left(\phi_{n, k} ; \mu_{n, k}^{(j)}, \Lambda_{k}^{(j)}\right) .
$$

\section{REFERENCES}

[1] J. A. C. Bingham, "Multicarrier modulation for data transmission: an idea whose time has come," IEEE Communications Magazine, vol. 28, no. 5, pp. 5-14, 1990.

[2] Z. Wang and G. B. Giannakis, "Wireless multicarrier communications," IEEE Signal Processing Magazine, vol. 17, no. 3, pp. 29-48, 2000.

[3] T. Pollet, M. van Bladel, and M. Moeneclaey, "BER sensitivity of OFDM systems to carrier frequency offset and Wiener phase noise," IEEE Transactions on Communications, vol. 43, no. 234, pp. 191-193, 1995.
[4] E. Costa and S. Pupolin, "M-QAM-OFDM system performance in the presence of a nonlinear amplifier and phase noise," IEEE Transactions on Communications, vol. 50, no. 3, pp. 462-472, 2002.

[5] L. Tomba, "On the effect of Wiener phase noise in OFDM systems," IEEE Transactions on Communications, vol. 46, no. 5, pp. 580-583, 1998.

[6] L. Tomba and W. A. Krzymien, "Sensitivity of the MC-CDMA access scheme to carrier phase noise and frequency offset," IEEE Transactions on Vehicular Technology, vol. 48, no. 5, pp. 1657-1665, 1999.

[7] H. Steendam and M. Moeneclaey, "The effect of carrier phase jitter on MC-DS-CDMA," in Proceedings of the IEEE International Conference on Communications (ICC '01), vol. 6, pp. 1881-1884, Helsinki, Finland, June 2001.

[8] C. Garnier, L. Clavier, Y. Delignon, M. Loosvelt, and D. Boulinguez, "Multiple acces for $60 \mathrm{GHz}$ mobile ad hoc networks," in Proceedings of the 55th IEEE Vehicular Technology Conference (VTC '02), vol. 3, pp. 1517-1521, Birmingham, Ala, USA, May 2002.

[9] R. A. Casas, S. L. Biracree, and A. E. Youtz, "Time domain phase noise correction for OFDM signals," IEEE Transactions on Broadcasting, vol. 48, no. 3, pp. 230-236, 2002.

[10] P. Robertson and S. Kaiser, "Analysis of the effects of phase noise in orthogonal frequency division multiplexing (OFDM) systems," in Proceedings of the IEEE International Conference on Communications (ICC '95), pp. 1652-1657, Seattle, Wash, USA, June 1995.

[11] D. Yee, J. R. Reilly, and T. Kirubarajan, "Channel equalization and phase noise suppression for OFDM systems in a time-varying frequency channel using particle filtering," in Proceedings of the IEEE International Conference on Acoustics, Speech, and Signal Processing (ICASSP '05), vol. 3, pp. 777-780, Philadelphia, Pa, USA, March 2005.

[12] D. Petrovic, W. Rave, and G. Fettweis, "Common phase error due to phase noise in OFDM-estimation and suppression," in Proceedings of the 15th IEEE International Symposium on Personal, Indoor and Mobile Radio Communications (PIMRC '04), vol. 3, pp. 1901-1905, Barcelona, Spain, September 2004.

[13] S. Wu and Y. Bar-Ness, "OFDM systems in the presence of phase noise: consequences and solutions," IEEE Transactions on Communications, vol. 52, no. 11, pp. 1988-1996, 2004.

[14] D. Petrovic, W. Rave, and G. Fettweis, "Intercarrier interference due to phase noise in OFDM-estimation and suppression," in Proceedings of the 60th IEEE Vehicular Technology Conference (VTC '04), vol. 3, pp. 2191-2195, Los Angeles, Calif, USA, September 2004.

[15] S. Wu and Y. Bar-Ness, "A new phase noise mitigation method in OFDM systems with simultaneous CPE and ICI correction," in Proceedings of the 4th International Workshop Multi-Carrier Spread Spectrum (MCSS '03), pp. 501-508, Oberpfaffenhofen, Germany, September 2003.

[16] K. Nikitopoulos and A. Polydoros, "Phase-impairment effects and compensation algorithms for OFDM systems," IEEE Transactions on Communications, vol. 53, no. 4, pp. 698-707, 2005.

[17] D. D. Lin and T. J. Lim, "The variational inference approach to joint data detection and phase noise estimation in OFDM," IEEE Transactions on Signal Processing, vol. 55, no. 5, pp. 18621874, 2007.

[18] A. Doucet, N. de Freitas, and N. Gordon, Sequential Monte Carlo Methods in Practice, Springer, New York, NY, USA, 2001. 
[19] N. Dinur and D. Wulich, "Peak-to-average power ratio in high-order OFDM," IEEE Transactions on Communications, vol. 49, no. 6, pp. 1063-1072, 2001.

[20] N. Chen, G. T. Zhou, and H. Qian, "Power efficiency improvements through peak-to-average power ratio reduction and power amplifier linearization," EURASIP Journal on Advances in Signal Processing, vol. 2007, Article ID 20463, 7 pages, 2007.

[21] S. Wei, D. L. Goeckel, and P. A. Kelly, "The complex enveloppe of a bandlimited OFDM signal converges weakly to a Gaussian random process: proof and application," to appear in IEEE Transactions on Information Theory, http://www.ece.lsu.edu/swei.

[22] F. Septier, Y. Delignon, A. Menhaj-Rivenq, and C. Garnier, "Monte Carlo methods for channel, phase noise, frequence offset estimation with unknwon noise variances in OFDM systems," to appear in IEEE Transactions on Signal Processing.

[23] B. Anderson and J. Moore, Optimal Filtering, Prentice-Hall, Englewood Cliffs, NJ, USA, 1979.

[24] M. S. Arulampalam, S. Maskell, N. Gordon, and T. Clapp, "A tutorial on particle filters for online nonlinear/non-Gaussian Bayesian tracking," IEEE Transactions on Signal Processing, vol. 50, no. 2, pp. 174-188, 2002.

[25] J. S. Liu and R. Chen, "Sequential Monte Carlo methods for dynamic systems," Journal of the American Statistical Association, vol. 93, no. 443, pp. 1032-1044, 1998.

[26] M. Bolić, P. M. Djurić, and S. Hong, "New resampling algorithms for particle filters," in Proceedings of the IEEE International Conference on Acoustics, Speech and Signal Processing (ICASSP '03), vol. 2, pp. 589-592, Hong Kong, April 2003.

[27] J. Hol, T. Schön, and F. Gustafsson, "On resampling algorithms for particle filters," in Proceedings of the Nonlinear Statistical Signal Processing Workshop (NSSPW '06), Cambridge, UK, September 2006.

[28] M. Bolić, P. M. Djurić, and S. Hong, "Resampling algorithms for particle filters: a computational complexity perspective," EURASIP Journal on Applied Signal Processing, vol. 2004, no. 15, pp. 2267-2277, 2004.

[29] R. Chen and J. S. Liu, "Mixture Kalman filters," Journal of the Royal Statistical Society B, vol. 62, no. 3, pp. 493-508, 2000.

[30] T. Schön, F. Gustafsson, and P.-J. Nordlund, "Marginalized particle filters for mixed linear/nonlinear state-space models," IEEE Transactions on Signal Processing, vol. 53, no. 7, pp. 2279_ 2289, 2005.

[31] G. Storvik, "Particle filters for state-space models with the presence of unknown static parameters," IEEE Transactions on Signal Processing, vol. 50, no. 2, pp. 281-289, 2002.

[32] V. Zaritskii, V. Svetnik, and L. Shimelevich, "Monte Carlo technique in problems of optimal data processing," Automation and Remote Control, vol. 12, pp. 95-103, 1975.

[33] H. L. van Trees, Detection, Estimation and Modulation Theory, John Wiley \& Sons, New York, NY, USA, 1968.

[34] P. Tichavsky, "Posterior Cramér-Rao bound for adaptive harmonic retrieval," IEEE Transactions on Signal Processing, vol. 43, no. 5, pp. 1299-1302, 1995.

[35] P. Tichavsky, C. H. Muravchik, and A. Nehorai, "Posterior Cramér-Rao bounds for discrete-time nonlinear filtering," IEEE Transactions on Signal Processing, vol. 46, no. 5, pp. 13861396, 1998.

[36] T. Hrycak and G. Matz, "Low-complexity time-domain ICI equalization for OFDM communications over rapidly varying channels," in Proceedings of the 40th Asilomar Conference on Signals, Systems and Computers (ACSSC '06), pp. 1767-1771, Pacific Grove, Calif, USA, October-November 2006. 